\title{
Linguistic and content validation of the Patient-Generated Subjective Global Assessment (PG-SGA) translated and culturally adapted to the Italian setting
}

\author{
Ripamonti $\mathrm{Cl}^{1}$, Arena $\mathrm{F}^{2}$, Toffolatti L , Ferrara $\mathbf{M L}^{1}$, Guglielmo $\mathbf{M}^{1}$, Di Pede $\mathbf{P}^{1}$, Ottery FD ${ }^{3}$, \\ Laviano $\mathrm{A}^{4}$, Jager-Wittenaar $\mathrm{H}^{3,5}$, Bossi $\mathrm{P}^{6}$ \\ 1. Fondazione IRCCS Istituto Nazionale dei Tumori, Supportive Care in Cancer, Department Oncology-Hematology, Milano, Italy. \\ 2. University of Brescia, ASST Spedali Civili di Brescia, Chair of Hematology and Unit of Blood Disease and Stem Cell Transplantation, Department of Clinical And Experimental \\ Sciences, Brescia, Italy. \\ 3. Hanze University of Applied Sciences, Research Group Healthy Ageing, Allied Health Care and Nursing, Groningen, The Netherlands. \\ 4. Sapienza University, Clinical Medicine, Roma, Italy. \\ 5. University of Groningen, University Medical Center Groningen, Department of Maxillofacial Surgery, Groningen, The Netherlands. \\ 6. Fondazione IRCCS Istituto Nazionale dei Tumori, Head \& Neck Complex Unit- Department Oncology-Hematology, Milano, Italy.
}

\section{Objectives}

We aimed to test linguistic validity, i.e. perceived comprehensibility and difficulty, and content validity (relevance) of the Italian version of the PG-SGA in patients and a multidisciplinary sample of healthcare professionals.

\section{Introduction}

The Patient-Generated Subjective Global Assessment (PG-SGA) is an instrument to assess malnutrition and risk factors. The Boxes Weight, Intake, Symptoms and

Activities/Function are to be completed by the patient, and the Worksheets by the professional. We translated and culturally adapted the original PG-SGA for the Italian setting, according to ISPOR Principles.

\section{Methods}

- Linguistic validity of the Italian PG-SGA was assessed in 120 Italian cancer patients, and linguistic and content validity were assessed in 81 Italian professionnals (41 dietitians, 20 physicians, 18 medical students, 1 physiotherapist, 1 physiotherapy student).

- Comprehensibility, difficulty and content validity were operationalized by calculating item and scale indices.

- Scale indices 0.80-0.90 were considered acceptable, and indices $\geq 0.90$ were considered excellent.
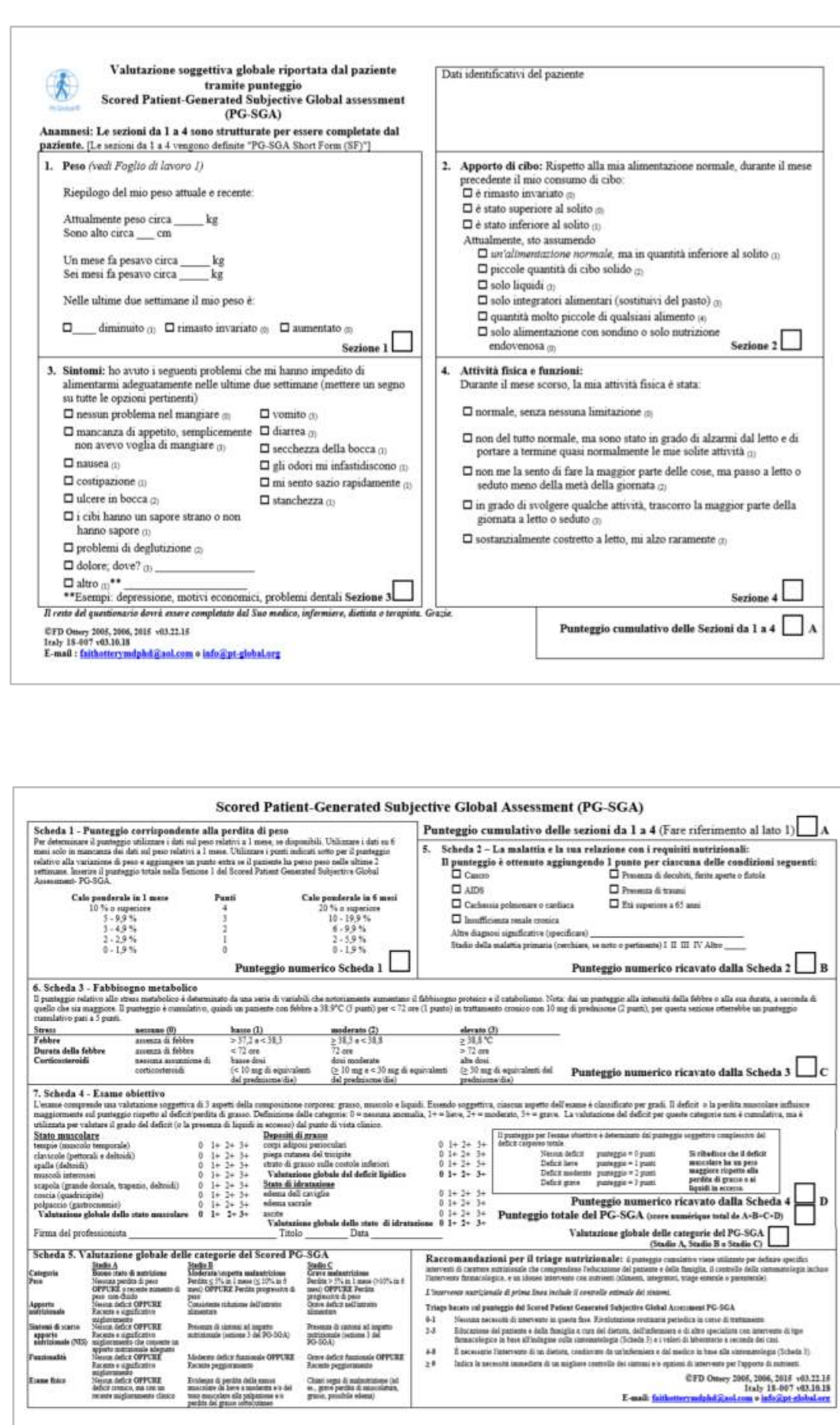

Figure 1. Italian version of the PG-SGA

\section{Results}

- Patients perceived comprehensibility and difficulty of the Boxes as excellent (S-Cl=0.98, S-DI=0.96).

- Professionals perceived comprehensibility of the Worksheets as excellent $(\mathrm{S}-\mathrm{Cl}=0.92)$, difficulty as acceptable (S-DI=0.85), and content validity of the full PG-SGA as excellent (S-CVI=0.92).

- Dietitians gave higher scores on comprehensibility, difficulty and content validity of Worksheet 4 (physical exam) than the other professions.

- In Worksheet 4, the temples, clavicles, shoulders, interosseous muscles, scapula, and orbital fat pads were considered most difficult to evaluate.

\section{Conclusion}

Translation and cultural adaptation of the PG-SGA resulted in a Italian version that maintained its original purpose and meaning, and can be completed adequately by patients and professionals. 